\title{
Quick starting hormonal contraception after using oral emergency contraception: a systematic review
}

\author{
Lauren EE Murphy, ${ }^{1,2}$ Zhong E Chen, ${ }^{3}$ Valerie Warner, ${ }^{3}$ \\ Sharon T Cameron ${ }^{1}$
}

${ }^{1}$ Department of Reproductive and Developmental Sciences, University of Edinburgh, Edinburgh, UK

${ }^{2}$ Brighton and Sussex Medical School, University of Sussex, Brighton, UK

${ }^{3}$ Chalmers Centre, Edinburgh, UK

\section{Correspondence to} Lauren EE Murphy, 2A Chalmers Street, Edinburgh EH3 9ES, UK; I. murphy1@uni.bsms.ac.uk

Received 25 January 2017 Revised 28 March 2017 Accepted 29 May 2017 Published Online First 29 June 2017

\section{CrossMark}

To cite: Murphy LEE, Chen ZE, Warner V, et al. J Fam Plann Reprod Health Care 2017:43:319-326.

\begin{abstract}
Introduction Unprotected intercourse after oral emergency contraception (EC) significantly increases pregnancy risk. This underlies the importance of promptly starting effective, ongoing contraception - known as 'quick starting'. However, theoretical concern exists that quick starting might interact with $\mathrm{EC}$ or hormonal contraception ( $\mathrm{HC}$ ) potentially causing adverse side effects.

Method A systematic review was conducted, evaluating quick starting $\mathrm{HC}$ after oral $\mathrm{EC}$ [levonorgestrel $1.5 \mathrm{mg}$ (LNG) or ulipristal acetate $30 \mathrm{mg}$ (UPA)]. PubMed, EMBASE, The Cochrane Library, ICTRP, ClinicalTrials.gov and relevant reference lists were searched in February 2016. A lack of comparable studies prevented metaanalysis.

Results Three randomised controlled trials were identified. Two biomedical studies suggested HC action was unaffected by quick starting after UPA; one study examined ovarian quiescence (OR 1.27; 95\% Cl 0.51-3.18) while taking combined oral contraception (COC). Another assessed cervical mucus impenetrability (OR 0.76; $95 \% \mathrm{Cl}$ $0.27-2.13$ ) while taking progestogen-only pills (POP). Quick starting POP reduced the ability of UPA to delay ovulation (OR 0.04; $95 \% \mathrm{Cl}$ $0.01-0.37$ ). Side effects (OR 1.22; $95 \% \mathrm{Cl}$ $0.48-3.12)$ and unscheduled bleeding (OR 0.53; $95 \% \mathrm{Cl}$ 0.16-1.81) were unaffected by quick starting COC after UPA. Another study reported higher self-reported contraceptive use at 8 weeks among women quick starting POP after LNG, compared with women given LNG alone (OR 6.73; 95\% Cl 2.14-21.20).
\end{abstract}

\section{INTRODUCTION}

Oral emergency contraception (EC), levonorgestrel $1.5 \mathrm{mg}$ (LNG) or ulipristal acetate $30 \mathrm{mg}$ (UPA), can prevent

\section{Key message points}

The contraceptive action of the combined hormonal contraceptive pill or progestogen-only pill (POP) is not reduced when quick started after using ulipristal acetate (UPA).

- The ability of UPA to delay ovulation is reduced when a POP (desogestrel) is quick started 1 day after using UPA.

- Adverse effects (including unscheduled bleeding) are not increased when combined hormonal contraception is quick started after using UPA.

pregnancy following unprotected sexual intercourse (UPSI). ${ }^{1}$ A Cochrane review found that further UPSI in the same cycle as EC increased pregnancy rates two to three times compared with women who did not have sex after taking EC. ${ }^{2}$ This raises the importance of initiating regular contraception immediately after oral EC rather than waiting until the next menstrual cycle - a practice known as 'quick starting'.3

Quick starting hormonal contraception (HC) after UPA may decrease either drug's effectiveness since UPA, a progesterone receptor modulator (PRM), may counteract $\mathrm{HC}$ action, potentially causing side effects. ${ }^{45}$ Effectiveness and side effects influence contraceptive use, making it important to assess the impact of quick starting $\mathrm{HC}$ after EC. ${ }^{6}$ Although LNG is unlikely to interact with HCs, since several contain LNG, quick starting could theoretically cause side effects and affect ongoing contraceptive use. ${ }^{7}$ This review aims to assess the impact of quick starting HC after oral EC, plus resultant side effects and ongoing contraceptive use. 


\section{METHODS}

A review protocol (CRD42016033170) was registered with PROSPERO, an international prospective register of systematic reviews (UK). ${ }^{8}$

\section{Literature source}

In February 2016, two reviewers (LM and VW) independently searched PubMed, EMBASE, The Cochrane Library, ClinicalTrials.gov and The International Clinical Trials Registry Platform (ICTRP) for relevant studies (from database inception through to 18 February 2016). The search strategies were as follows:

EMBASE/Pubmed/The Cochrane Library: ('quick start"' OR quick-start* OR 'immediate start' OR quick-

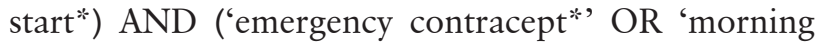
after' OR morning-after OR 'post coital' OR post-coital OR postcoital OR 'unprotected sexual intercourse' OR 'unprotected sex' OR 'unprotected intercourse' OR 'unsafe sex' OR 'ulipristal acetate' OR UPA OR ellaOne OR 'progesterone receptor modulator"' OR levonorgestrel OR LNG OR levonelle) AND (contracept" OR 'contraceptive agent"' OR 'hormonal contracept"').

ClinicalTrials.gov and ICTRP: Condition: emergency contraception; Intervention: (levonorgestrel OR ulipristal acetate) AND contraception.

Reference lists of relevant studies were independently reviewed to identify additional studies. Investigators were contacted for further details on published and unpublished studies.

\section{Eligibility of studies}

Articles were screened by title and abstract and compared with the predefined PICO (population, intervention, control and outcome) criteria, as described below, by two independent reviewers (LM and VW). If the relevance of the study was ambiguous, the full text was accessed and checked against the PICO criteria and study investigators were contacted by email. Advice was sought from a senior member of the research team (ZEC) when discrepancies occurred between the reviewers' results.

Randomised controlled trials (RCTs) and observational studies were included that matched the PICO questions. All women taking oral EC (UPA or LNG) and $\mathrm{HC}$ were included. Studies were included that quick started $\mathrm{HC}$ within 1 day of oral EC administration. We aimed to compare these participants to women who started $\mathrm{HC}$ at the beginning of the menstrual cycle following EC administration. Placebo-controlled trials were also included. The effectiveness of $\mathrm{HC}$ and EC, side effects, unscheduled bleeding and ongoing contraceptive use were assessed. Studies were not excluded based on publication status or language. Participants were not excluded based on prior HC use or their reason for requesting EC.
Assessment of study quality

Two reviewers (LM and VW) independently assessed study quality using GRADE (Grades of Recommendation, Assessment, Development and Evaluation) protocol. ${ }^{9}$ Details of the included studies are presented in Tables 1 and 2, collating the data for each outcome.

\section{Statistical analysis}

For dichotomous results, odd ratios (ORs) and 95\% confidence intervals (CIs) were calculated. An absolute risk and risk difference were then calculated using a control baseline risk measure, taken from a highquality observational study. ${ }^{9}$ In trials with multiple study arms, only the subset of data matching the PICO criteria was used. Meta-analysis was planned, however data could not be combined due to differences in study design.

\section{RESULTS}

Sixty-one unduplicated papers were identified, as shown in Figure 1. After screening, 43 full-text articles were examined. Reviews were not excluded at this stage to allow reference lists to be checked. Three RCTs were included in this systematic review. No trials were combined for meta-analysis due to a lack of similar study design.

\section{Included studies}

Three RCTs met the inclusion criteria. ${ }^{10-12}$ Two studies administering UPA were conducted in Edinburgh (UK) by one research team. ${ }^{11}{ }^{12}$ Other sites included Stockholm (Sweden), Groningen (Netherlands) ${ }^{11}$ and Santo Domingo (Dominican Republic). ${ }^{10}$ Two of the studies were biomedical studies that recruited healthy volunteers who had not had UPSI. ${ }^{10} 11$ The remaining cluster randomised trial recruited pharmacies that enrolled women presenting for EC (LNG) after UPSI. ${ }^{12}$

Overall, 293 women were included. Sample sizes ranged from $49^{10}$ to $168 .{ }^{12}$ Prior $\mathrm{HC}$ use differed between studies. Two studies, including the study of women presenting after UPSI, excluded individuals using $\mathrm{HC}$ at study enrolment ${ }^{10}{ }^{12}$ A further study excluded healthy volunteers who used a progestogen-only method within 3 months of study enrollment but included women using combined oral contraception (COC). ${ }^{11}$

One study compared 21 days of COC [Microgynon $30^{\circledR}$ containing $30 \mu \mathrm{g}$ ethinylestradiol (EE) and $150 \mu \mathrm{g}$ LNG]) use, administered within 1 day of UPA, with 21 days of COC use initiated 1 day after a placebo in healthy volunteers. ${ }^{11}$ In this study, UPA/ placebo administration took place once an ovarian follicle was $>13 \mathrm{~mm}$ in diameter on a transvaginal ultrasound scan (TVU), which corresponds to the mid-to-late follicular phase of the menstrual cycle. ${ }^{11}$ Another study administered either UPA or placebo, followed 1 day later by either 20 days of progestogen-only pill (POP) [Cerazette ${ }^{\circledR}$ containing $75 \mu \mathrm{g}$ desogestrel (DSG)] or 20 days of a placebo to healthy 


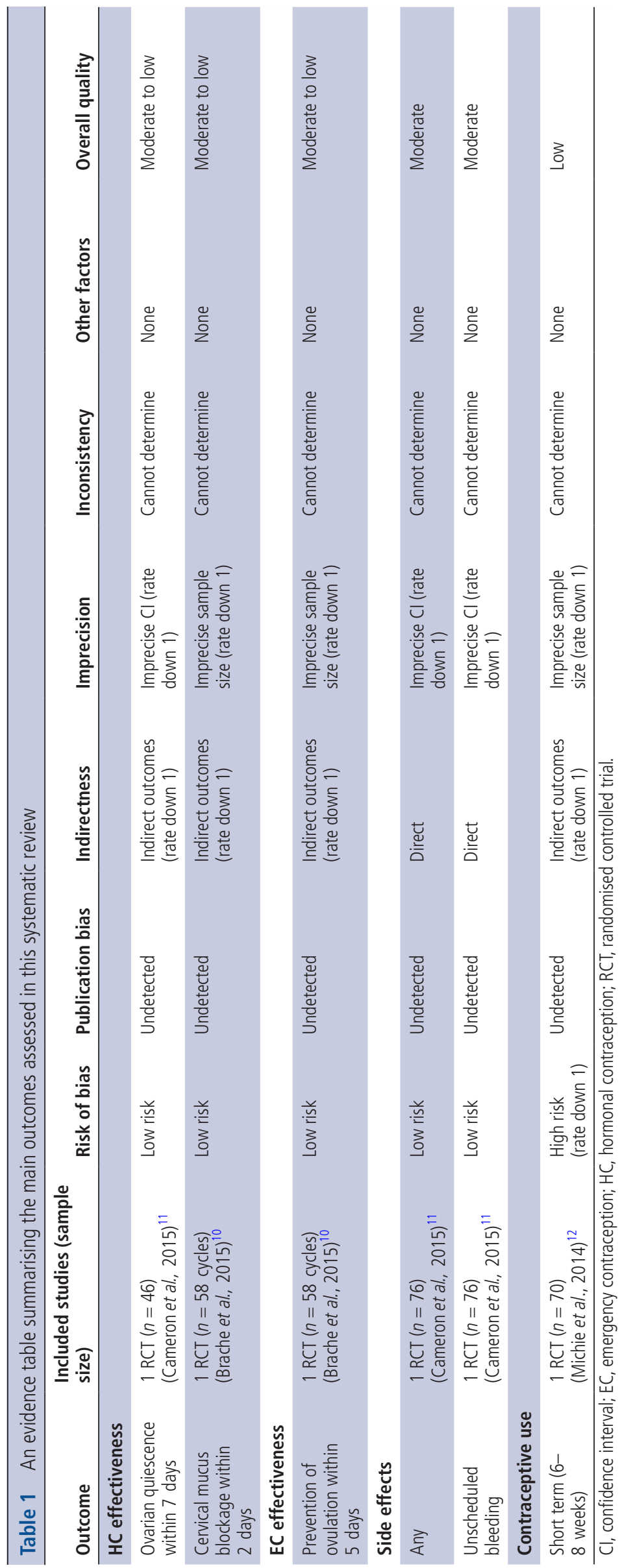




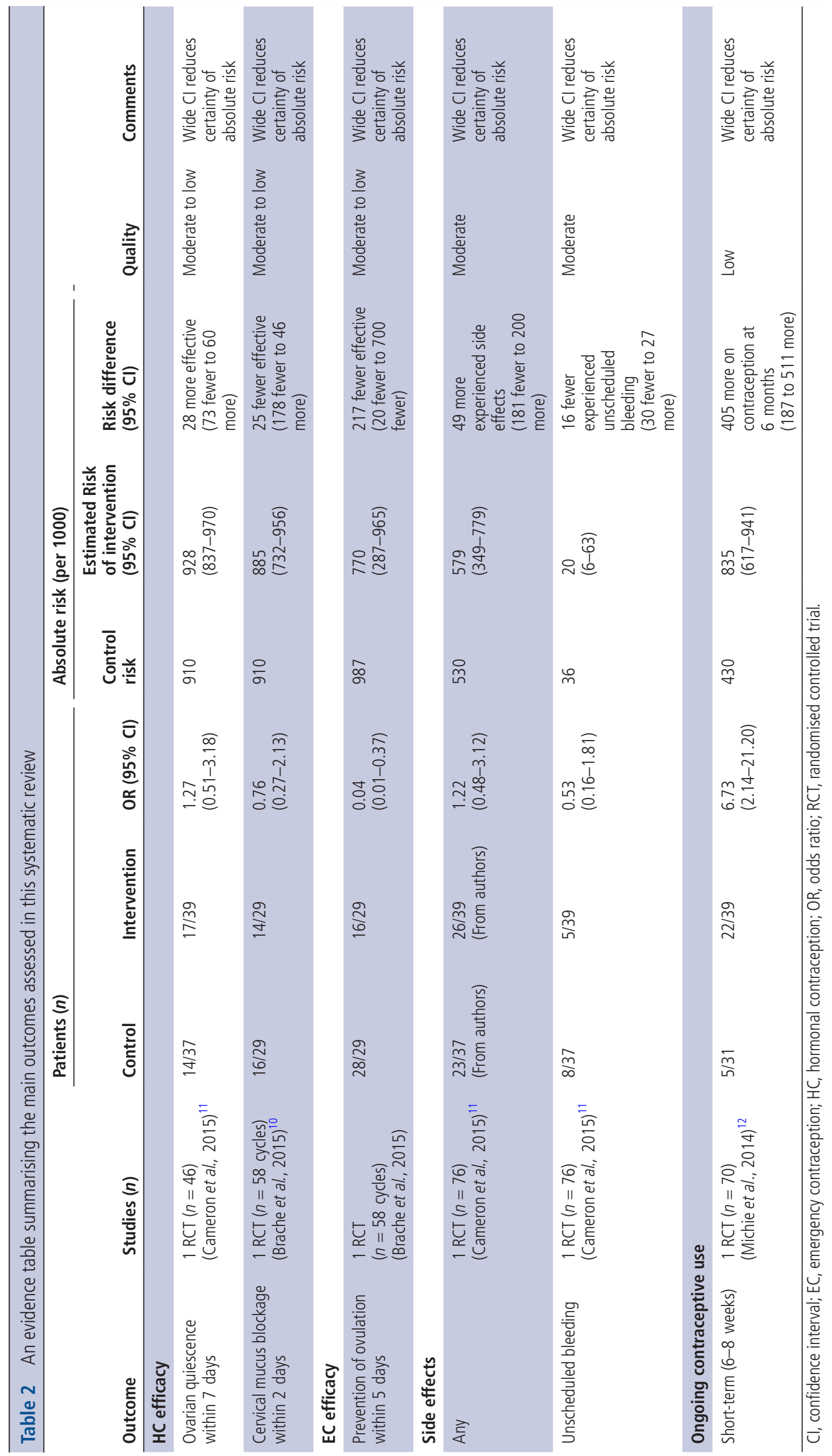




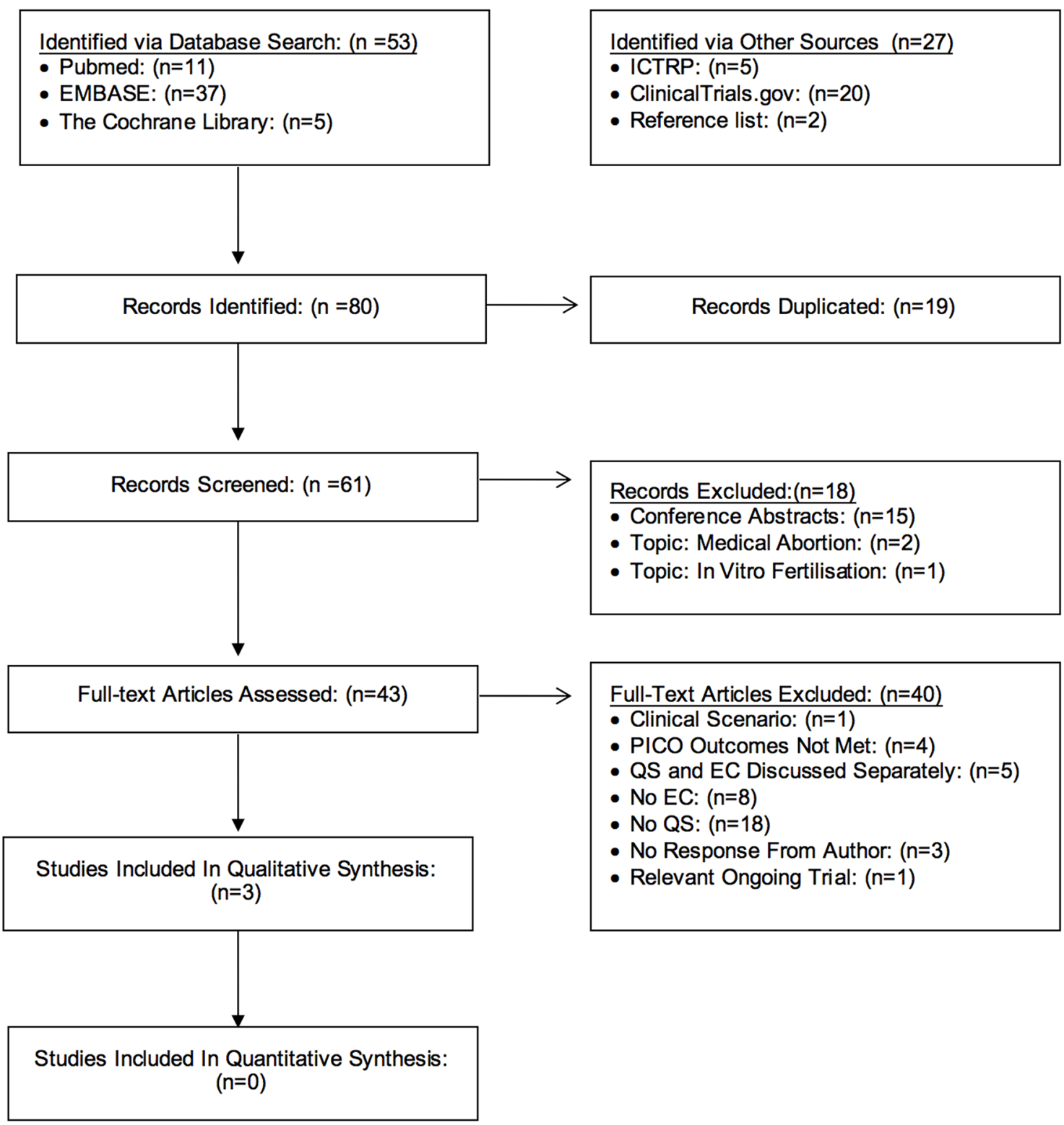

Figure 1 A flow diagram depicting how studies were identified and selected for inclusion in a systematic review on quick starting (QS) hormonal contraception after emergency contraception (EC).

volunteers. ${ }^{10}$ The UPA/placebo was administered when the lead ovarian follicular diameter was $\geq 14 \mathrm{~mm}$, corresponding to the mid-to-late follicular phase of the menstrual cycle. ${ }^{10} \mathrm{~A}$ partial crossover design was employed with each participant receiving two different treatment combinations with one menstrual cycle in between. In the third study, LNG was administered to all women who presented to a pharmacy after UPSI. ${ }^{12}$ Women then received either 35 days of low-dose POP (Norgeston ${ }^{\circledR}$ containing $35 \mu \mathrm{g}$ LNG), a rapid access appointment at a family planning clinic to discuss and initiate contraception, or standard care (i.e. verbal and/or written advice on where contraception could be obtained). Women were followed up by telephone after 6-8 weeks. Information on when women in the rapid access group started contraception was not obtained so this trial arm was excluded from our analysis.
Measures of $\mathrm{HC}$ effectiveness when quick starting HC after EC use were assessed by two studies, although neither measured pregnancy rates since volunteers were instructed to abstain from sex or use barrier contraceptives throughout the trials. ${ }^{10} 11$ One RCT used TVU measurements of follicle size and serum estradiol/progesterone levels to calculate Hoogland scores of ovarian activity after UPA administration and COC (Microgynon 30 containing $30 \mu \mathrm{g} \mathrm{EE}$ and $150 \mu \mathrm{g}$ LNG) use; scores of three or less indicated ovarian quiescence. ${ }^{11}$ Another study determined if the POP (Cerazette containing $75 \mu \mathrm{g}$ DSG) had exerted contraceptive effects on cervical mucus after UPA use by calculating a modified World Health Organizaion Cervical Mucus Score; scores of four or less indicated that mucus had become impenetrable to sperm. ${ }^{10}$ This study also gathered TVU measurements of follicle size, 
plasma etonogestrel levels (the active metabolite of desogestrel) and serum estradiol/progesterone levels. These latter data were not shown and we were unable to obtain these data from the authors. The study reported no differences in etonogestrel levels between groups.

The efficacy of UPA was assessed indirectly in one study by measuring ovulation rates within 5 days of UPA administration, with concurrent POP (Cerazette containing $75 \mu \mathrm{g}$ DSG) use. $^{10}$ UPA typically delays ovulation by at least 5 days, during which time sperm in the female reproductive tract would be non-viable. ${ }^{13}$ Ovulation was judged to have occurred in this study if TVU identified a follicular rupture and serum progesterone levels were $\geq 10 \mathrm{nmol} / \mathrm{L}$ on two consecutive visits. $^{10}$

Participants quick starting COC (Microgynon 30 containing $30 \mu \mathrm{g}$ EE and $150 \mu \mathrm{g}$ LNG) after UPA or placebo in one biomedical study reported adverse effects and unscheduled bleeding during the 21 days follow-up. ${ }^{11}$

In the cluster randomised trial of POP (Norgeston containing $35 \mu \mathrm{g}$ LNG) use after EC LNG, contraceptive use was self-reported by women at $6-8$ weeks. ${ }^{12}$

\section{Excluded studies}

Seven studies were excluded. ${ }^{14-20}$ Three of these did not assess how many women quick started HC after EC. ${ }^{161819}$ Two studies were excluded since there was no follow-up. ${ }^{1420}$ In one study, it was unclear if participants had initiated HC within 1 day of oral EC administration. ${ }^{17}$ In another study, it was unclear which of the outcomes included women who had quick started HC after EC. ${ }^{15}$ One ongoing trial at the recruitment phase was identified. $^{21}$

\section{Quality of included studies}

A summary of the study quality across each outcome is shown in Table 1. Each RCT was rated as low, moderate-to-low or moderate for overall quality. No observational studies were included in this review so dose-response, large effect size and the impact of confounding factors on the results were not assessed.

Two studies adequately randomised and concealed participant allocation so the risk of confounding bias was deemed low. ${ }^{10} 11$ One trial used restricted randomisation to randomise pharmacies and had no allocation concealment, making this RCT high risk for confounding bias. ${ }^{12}$ Investigators, participants and sponsors were blinded in one study, making it low risk for allocation bias. ${ }^{11}$ One biomedical study was singleblinded, which may have affected outcome measures since investigators were aware of allocation. ${ }^{10}$ In the cluster randomised trial no blinding occurred, resulting in a high risk of bias. ${ }^{12}$ An intention-to-treat approach was utilised by all studies. ${ }^{10-12}$ Two studies had acceptable lost-to-follow-up figures of $<20 \%$ and were thus at a low risk of attrition bias. ${ }^{10} 11$ A third study had a lost-to-follow-up of 39\% and so the quality was rated down. ${ }^{12}$

Selective reporting was not identified in two studies since their protocols were available and examined on ClinicalTrials.gov. ${ }^{11} 12$ The risk of reporting bias was unclear in another study since the protocol could not be obtained. ${ }^{10}$

Several outcome measures were indirect. For HC and EC effectiveness, the biomedical studies used surrogate measures of Hoogland scores, cervical mucus scores and ovulation rates. ${ }^{10} 11$ Short-term HC use was measured in the one cluster randomised trial as self-reported contraceptive use within 6-8 weeks of LNG use. ${ }^{12}$ Outcome measures for side effects and unscheduled bleeding directly matched the PICO question. ${ }^{11}$

Two studies did not meet their predefined sample sizes and were rated down for imprecision, despite having narrow CIs for EC effectiveness and ongoing HC use. $^{10}{ }^{12}$ Another study had an adequate sample size but all the outcome measures, including HC effectiveness, unscheduled bleeding and side effects had wide CIs, making the results imprecise. ${ }^{11}$

Inconsistency could not be determined as only one study was included per outcome. However, no relevant, unpublished studies were identified from the databases and each trial had different results, making it seem unlikely that publication bias had occurred. ${ }^{9}$

\section{Quantitative results}

The overall results for each outcome are shown in Table 2. HC effectiveness was assessed by ovarian quiescence rates within 7 days (OR 1.27; 95\% CI $0.51-3.18)$ in one study of women receiving either UPA or placebo, followed by COC (Microgynon 30 containing $30 \mu \mathrm{g} \mathrm{EE}$ and $150 \mu \mathrm{g} \mathrm{LNG}) .{ }^{11}$ One biomedical study assessed HC effectiveness when women received UPA or placebo and then initiated POP (Cerazette containing $75 \mu \mathrm{g}$ DSG), by determining cervical mucus scores within 2 days of UPA/ placebo use (OR 0.76; 95\% CI 0.27-2.13). ${ }^{10}$ These timeframes reflect the time taken for contraceptive effectiveness to be achieved when initiating COC or POP. $^{22}{ }^{23}$ Both studies stated that there was no significant difference in $\mathrm{HC}$ effectiveness between women quick starting HC after UPA and women using only HC. However, when the ORs were calculated for this review, the CIs in both studies were wide, making the results imprecise.

EC effectiveness was measured in one study, based on ovulation rates within 5 days of UPA use, during which time women were taking POP (Cerazette containing $75 \mu \mathrm{g}$ DSG) or placebo (OR 0.04; 95\% CI $0.01-0.37) .{ }^{10}$ The study reported that quick starting POP reduced UPA effectiveness compared with using UPA alone.

Side effects (OR 1.22, 95\% CI $0.48-3.12$ ) and unscheduled bleeding (OR 0.53; 95\% CI $0.16-$ 1.81) were assessed in women quick starting COC 
(Microgynon 30 containing $30 \mu \mathrm{g} \mathrm{EE}$ and $150 \mu \mathrm{g}$ LNG) after UPA or placebo administration. ${ }^{11}$ This found that quick starting COC after UPA was non-inferior to taking COC alone for both outcomes. ${ }^{11}$ However, when the ORs were calculated in this review from data gathered via author communication, both outcomes had wide CIs, making the results less precise. Self-reported contraceptive use 6-8 weeks after quick starting a POP (Norgeston containing $35 \mu \mathrm{g}$ LNG) after EC LNG was higher among women receiving a 1-month supply of the POP compared with the group receiving only advice on where to obtain contraceptive supplies (OR 6.73; 95\% CI 2.14-21.20), although the small sample size and wide CIs reduced the precision. ${ }^{12}$

\section{DISCUSSION}

Limited evidence identified in this systematic review suggests that quick starting HC after UPA does not reduce $\mathrm{HC}$ efficacy or increase the risk of side effects or unscheduled bleeding. ${ }^{10}{ }^{11}$ However, starting a POP immediately after UPA reduces the ability of UPA to delay ovulation. ${ }^{10}$ Limited evidence suggests that quick starting a POP after EC LNG increases rates of self-reported $\mathrm{HC}$ use at 6-8 weeks, compared with when no POP was immediately supplied. ${ }^{12}$

The Faculty of Sexual \& Reproductive Healthcare (FSRH) currently recommend women wait at least 5 days after UPA use before initiating HC. ${ }^{24}$ Additional contraceptive precautions (i.e. consistent condom use and/or abstinence) are required until HC effectiveness is established. This recommendation is based on Brache et al. (2015) included in this systematic review, which demonstrated that ovulation rates within 5 days of UPA use were higher among women who quick started a DSG-containing POP compared with women using a placebo. ${ }^{10} \mathrm{~A}$ previous biomedical study of healthy volunteers taking a DSG-containing POP with intermittent administration of a placebo or another PRM (Org 31710), showed that 29 \% more women ovulated in the PRM group, supporting the concern that a POP may alter the efficacy of a PRM used for EC, such as UPA. ${ }^{25}$ In the absence of further evidence, it is recommended that women delay initiating HC after UPA to ensure the efficacy of UPA is not compromised. The findings from an ongoing trial evaluating the impact of COC on UPA when the former is quick started will further clarify if quick starting a COC impacts on the ability of UPA to delay ovulation, although further research is needed. ${ }^{21}$ No studies assessed if quick starting HC after EC LNG use would alter the efficacy of EC LNG and/or HC, although the presence of LNG in HCs suggest that an interaction is unlikely. $^{7}$

EC is available in the UK and most other European countries without a prescription and increasingly women are choosing to access EC from this setting. ${ }^{26}$ Yet the pharmacy is usually unable to provide ongoing contraception. Provision of a bridging method of contraception such as the POP with EC has been shown to be feasible. ${ }^{12}$ Michie et al. (2014), included in this systematic review, found that this improved self-reported HC use at 6-8 weeks. ${ }^{12}$. One study found that $55 \%$ of women who could have quick started HC after EC did not return to the clinic within 6 months, potentially putting them at a higher risk of pregnancy. ${ }^{20}$ Studies have also shown that EC users are keen to quick start HC after EC use, with $89.5 \%$ of women in one survey wishing to start effective contraception after EC. ${ }^{14}$ However, without large robust trials it is uncertain whether quick starting leads to long-term contraceptive use and thus its impact on reducing unintended pregnancy rates.

There are limitations to this systematic review. The evidence quality for each outcome in this review was rated as low, moderate-to-low or moderate, since only one RCT was identified for each outcome. The lack of direct measures of contraceptive effectiveness, that is, pregnancy rates, and direct comparisons to women initiating $\mathrm{HC}$ in the next menstrual cycle further limited the results. Moreover, the findings cannot be generalised to all $\mathrm{HC}$ forms since only three oral HCs were included across the studies. Two out of three small studies were biomedical studies involving healthy volunteers, which may not reflect real world clinical practice and findings, particularly the impact quick starting HC has on UPA efficacy and therefore pregnancy risk. ${ }^{10} 11$

In conclusion, this is the only systematic review to date that examines the effectiveness and impact on bleeding patterns and side effects of quick starting HC after using oral EC. Women should be advised that further UPSI in the same cycle after EC use is associated with an increased risk of pregnancy compared with women who do not have further sex in that menstrual cycle, hence the importance of starting an effective method of contraception as soon as possible. Findings from this systematic review support the FSRH recommendation that women should wait at least 5 days after using UPA before initiating any $\mathrm{HC}$, alongside the additional days of abstinence and/or barrier method use for their chosen HC. ${ }^{24}$ Further large-scale research into the impact of quick starting HC after oral EC on the efficacy of either medication is required. To better inform public health policies, studies should also examine the impact of quick starting contraception after oral EC on unintended pregnancy rates and whether short-term provision of $\mathrm{HC}$ together with EC from a pharmacy would improve long-term continuation of effective contraception (e.g. at 12 months).

Contributors This study was undertaken as an undergraduate research project by LM, supervised by ZEC. LM produced and registered the protocol. LM and VW conducted the systematic literature search and assessed the included study quality. ZEC and SC reviewed and edited the drafts and final manuscript. 
Competing interests None declared.

Provenance and peer review Not commissioned; externally peer reviewed.

(C) Faculty of Sexual and Reproductive Healthcare of the Royal College of Obstetricians and Gynaecologists (unless otherwise stated in the text of the article) 2017. All rights reserved. No commercial use is permitted unless otherwise expressly granted.

\section{REFERENCES}

1 Faculty of Sexual \& Reproductive Healthcare (FSRH). Emergency Contraception, 2011. http://www.fsrh.org/ documents/ceu-emergency-contraception-jan-2012/. (accessed 14 Mar 2017).

2 Cheng L, Che Y, Gülmezoglu AM. Interventions for emergency contraception. Cochrane Database Syst Rev 2012;8:CD001324.

3 Faculty of Sexual \& Reproductive Healthcare (FSRH). Quick Starting Contraception, 2010. http://www.fsrh.org/documents/ ceuguidancequickstartingcontraception/. (accessed $14 \mathrm{Mar}$ 2017).

4 Gemzell-Danielsson K, Berger C, P G LL. Emergency contraception? mechanisms of action. Contraception 2013;87:300-8.

5 Smith OP, Critchley HO. Progestogen only contraception and endometrial break through bleeding. Angiogenesis 2005;8:11726.

6 Lessard LN, Karasek D, Ma S, et al. Contraceptive features preferred by women at high risk of unintended pregnancy. Perspect Sex Reprod Health 2012;44:194-200.

7 Royal Pharmaceutical Society. British National Formulary (BNF), 2016. https://www.medicinescomplete.com/mc/bnf/ current/. (accessed 14 Mar 2017).

8 National Institute for Health Research. PROSPERO International Prospective Register of Systematic Review. http://www.crd.york.ac.uk/PROSPERO/. (accessed 14 Mar 2017).

9 Guyatt G, Oxman AD, Akl EA, et al. GRADE guidelines: 1. Introduction-GRADE evidence profiles and summary of findings tables. J Clin Epidemiol 2011;64:383-94.

10 Brache V, Cochon L, Duijkers IJ, et al. A prospective, randomized, pharmacodynamic study of quick-starting a desogestrel progestin-only pill following ulipristal acetate for emergency contraception. Hum Reprod 2015;30:2785-93.

11 Cameron ST, Berger C, Michie L, et al. The effects on ovarian activity of ulipristal acetate when 'quickstarting' a combined oral contraceptive pill: a prospective, randomized, doubleblind parallel-arm, placebo-controlled study. Hum Reprod 2015;30:1566-72.

12 Michie L, Cameron ST, Glasier A, et al. Pharmacybased interventions for initiating effective contraception following the use of emergency contraception: a pilot study. Contraception 2014;90:447-53.

13 Brache V, Cochon L, Jesam C, et al. Immediate pre-ovulatory administration of $30 \mathrm{mg}$ ulipristal acetate significantly delays follicular rupture. Hum Reprod 2010;25:2256-63.

14 Baird AS, Trussell J, Webb A. Use of ulipristal acetate and levonorgestrel for emergency contraception: a follow-up study. J Fam Plann Reprod Health Care 2015;41:116-21.

15 Balkus J, Miller L. Same-day administration of depotmedroxyprogesterone acetate injection: a retrospective chart review. Contraception 2005;71:395-8.

16 Cameron ST, Glasier A, Johnstone A, et al. Ongoing contraception after use of emergency contraception from a specialist contraceptive service. Contraception 2011;84:36871.

17 Murthy AS, Creinin MD, Harwood B, et al. Same-day initiation of the transdermal hormonal delivery system (contraceptive patch) versus traditional initiation methods. Contraception 2005;72:333-6.

18 Nelson AL, Katz T. Initiation and continuation rates seen in 2-year experience with same day injections of DMPA. Contraception 2007;75:84-7.

19 Romero LM, Middleton D, Mueller T, et al. Improving the implementation of evidence-based clinical practices in adolescent reproductive health care services. J Adolesc Health 2015;57:488-95.

20 Simpson J, Craik J, Melvin L. Quick starting contraception after emergency contraception: have clinical guidelines made a difference? J Fam Plann Reprod Health Care 2014;40:184-9.

21 Edelman AB. Impact of combined hormonal contraceptives on UPA (registered study on ClinicalTrials.gov).

22 Faculty of Sexual \& Reproductive Healthcare (FSRH). Combined Hormonal Contraception. 2012 http://www.fsrh. org/documents/combined-hormonal-contraception/ (accessed 14 Mar 2017).

23 Faculty of Sexual \& Reproductive Healthcare (FSRH). Progestogen-only Pills. 2015 https://www.fsrh.org/standardsand-guidance/documents/ceuguidanceprogestogenonlypills/ (accessed 14 Mar 2017).

24 Faculty of Sexual \& Reproductive Healthcare (FSRH). CEU Statement: Quick Starting After UPA. 2015 http://www.fsrh. org/standards-and-guidance/documents/ceustatementquicksta rtingafterupa/ (accessed 14 Mar 2017).

25 Gemzell-Danielsson K, van Heusden AM, Killick SR, et al. Improving cycle control in progestogen-only contraceptive pill users by intermittent treatment with a new anti-progestogen. Hum Reprod 2002;17:2588-93.

26 Marston C, Meltzer H, Majeed A. Impact on contraceptive practice of making emergency hormonal contraception available over the counter in Great Britain: repeated cross sectional surveys. BMJ 2005;331:271. 